\title{
Comment on "Jamming at zero temperature and zero applied stress: The epitome of disorder"
}

\author{
Aleksandar Donev, ${ }^{1,2}$ Salvatore Torquato, ${ }^{1,2,3, *}$ Frank H. Stillinger, ${ }^{3}$ and Robert Connelly ${ }^{4}$ \\ ${ }^{1}$ Program in Applied and Computational Mathematics, Princeton University, Princeton NJ 08544 \\ ${ }^{2}$ Princeton Materials Institute, Princeton University, Princeton NJ 08544 \\ ${ }^{3}$ Department of Chemistry, Princeton University, Princeton NJ 08544 \\ ${ }^{4}$ Department of Mathematics, Cornell University, Ithaca NY 14853
}

\begin{abstract}
O’Hern, Silbert, Liu and Nagel [Phys. Rev. E. 68 , 011306 (2003)] (OSLN) claim that a special point $J$ of a "jamming phase diagram" (in density, temperature, stress space) is related to random close packing of hard spheres, and that it represents, for their suggested definitions of jammed and random, the recently introduced maximally random jammed state. We point out several difficulties with their definitions and question some of their claims. Furthermore, we discuss the connections between their algorithm and other hard-sphere packing algorithms in the literature.
\end{abstract}

Jammed random packings of hard particles have been and continue to be a subject of intense interest. The lack of precise definitions of both "jammed" and "random" have been a hindrance in the field, and recently efforts have emerged that have attempted to correct these deficiencies [1, 2, 3]. In particular, employing these new definitions, it has been shown that the venerable random close packed (RCP) state is ill-defined but can be replaced by the well-defined notion of a maximally random jammed (MRJ) state [1]. O'Hern, Silbert, Liu and Nagel (OSLN) [4] have recently written an interesting paper [4] that proposes a unified view of jamming for a variety of physical systems, including hard-sphere packings.

OSLN acknowledge the weaknesses of the conventional RCP state as stated in Ref. [1], but redefine MRJ (while still calling it RCP) by proposing new definitions for what constitutes a jammed and random system [5]. Given the subtlety of the problem, new definitions of "jammed" and "random" must be held to high mathematical standards in order to supplant existing ones. In this Comment, we question whether OSLN's "cleaner" definitions for these terms meet such standards. We also take issue with their claim to have generated unbiased and universal results of relevance to random sphere packings. Finally, we discuss the relationships between their algorithm and other packing algorithms.

\section{WHAT IS "JAMMED"?}

OSLN question whether the hard-sphere system is "physical" and therefore resort to studying particle systems with soft-sphere interactions to mimic hard-particle packings. The latter is inherently a geometrical problem. In fact, there is a simple and rigorous geometrical approach to jamming in hard-sphere systems that is not only well-defined, but, as we show below, is closely related to OSLN's jamming point $J$.

*Electronic address: torquato@electron.princeton.edu
Although the hard-sphere potential is an idealization, it is no less physical than any soft-sphere potential, especially in regards to jamming. Indeed, the singular nature of the hard-sphere potential is crucial because it enables one to be precise about the concept of "jamming." Recently, three hierarchically ordered jamming categories have been introduced [2]: local, collective and strict jamming. Each successive category progressively relaxes the boundary conditions imposed on the particle displacements. These definitions are very intuitive and completely geometric, and are closely linked to definitions of "rigid" or "stable" packings appearing in the mathematics literature [6, 7].

OSLN's definition of jamming simply states that the configuration of particles is at a stable (strict) energy minimum. Such a definition is dependent on the particular interparticle potential, and thus it obscures the relevant packing geometry (exclusion-volume effects). Furthermore, the distinctions between different jamming categories is critical, especially if one is trying to determine the density of the MRJ state [8]. Specifically, this density will generally be higher the more demanding is the jamming category. Clearly, OSLN do not distinguish between different degrees or levels of jamming. We have recently demonstrated that the distinction between collective and strict jamming is important even for very large packings, especially in two dimensions [8] .

For OSLN, a jammed configuration is one where there are no zero-frequency modes of the Hessian matrix of the total potential energy with respect to the positions of the particles (the dynamical matrix), while keeping the periodic unit cell fixed. Our definition of strict jamming relaxes this requirement and includes the lattice vectors as additional degrees of freedom [3]. As explained in detail in Ref. [9], the Hessian consists of two parts, a negative definite stress matrix and a positive semidefinite stiffness matrix. OSLN's definition of jammed means simply that the Hessian is positive definite at the energy minimum. A precise phrase for this is a stable or strict (local) energy minimum, and we see no point in redefining this elementary concept. In fact, according to OSLN, any stable energy minimum represents a jammed configuration, and 
it is not possible to relate this idea to packing concepts without numerous additional assumptions about the form of the pair potential and the interparticle distances at the energy minimum.

Although OSLN point out themselves that our definitions of jamming and MRJ are for hard-sphere packings, they claim to replace them with a "cleaner definition," which applies only to systems of soft spheres. The two definitions cannot directly be compared as they apply to different systems. However, OSLN themselves clearly imply that their "jammed" soft-sphere systems and "jammed" hard-sphere packings are related, by referring to other works on hard-sphere systems. For example, they claim a direct relation between their special point $J$ and RCP of hard spheres in Section IID of Ref. 4]. The basic idea, as OSLN explain, is that one "can approach the hard sphere by making the potential harder and harder...[to] produce a limiting hard sphere value". However, they question whether the hard-sphere limit is well-defined and "would argue that hard spheres are a singular limit and thus unphysical" and that "One should therefore concentrate on softer potentials for which unambiguous definitions can be constructed."

To demonstrate that the limit is well-defined, let us first define a collectively jammed sphere packing to be any nonoverlapping configuration of hard spheres in which no subset of particles can continuously be displaced so that its members move out of contact with one another and with the remainder set (while maintaining nonoverlap) [2]. The following theorem [9] shows that near the "jamming threshold" $\phi_{c}$, as defined in Section IIB in Ref. [4], the jamming of particle systems as defined by OSLN is directly related to this definition of collective jamming in hard-sphere packings:

Theorem: Consider an interparticle potential that is continuous and strictly monotonically decreasing around $r_{i j}=D$, and vanishes for $r_{i j}>D+\delta$. If in a finite configuration of particles interacting with such a potential, all interacting (i.e., closer then $D+\delta$ ) particles are a distance $D$ apart, and the configuration is a stable local energy minimum, then the configuration corresponds to a collectively jammed packing of hard spheres with diameter $D$.

If one relaxes the condition that all interacting particles are exactly at distance $D$ apart and instead asks only that the minimum interparticle distance be $D$, then for a sufficiently small $\delta$ one can prove [10] that the above sphere packing is almost collectively jammed (i.e., it is trapped in a small neighborhood of the initial configuration [3]). This theorem implies that the packings studied in Ref. 44 that are very slightly above the "jamming threshold" $\phi_{c}$ are indeed closely related to collectively jammed ideal packings of spheres of diameter $D=\sigma$ (polydispersity is trivial to incorporate). All of these considerations call into question the value of a definition of jamming that hinges on eigenvalues of dynamical matrices.

Finally, it is important to note that despite the fact that our definition of collective jamming above calls for virtual displacing (groups of) particles, one can in fact rigorously test for our hard-particle jamming categories using linear programming [3], without what OSLN call "shifting particles," even for very large disordered packings [8]. We have in fact communicated to OSLN the results 11] of our algorithm applied to several sample packings provided by them. In short, our algorithm verified that OSLN's systems near $\phi_{c}$ were indeed nearly collectively jammed (within a very small tolerance) when viewed as packings. However, they were not strictly jammed because OSLN keep the lattice vectors fixed during energy minimization.

\section{WHAT IS "RANDOM"?}

We agree that the maximum of an appropriate "entropic" metric would be a potentially useful way to characterize the randomness of a packing and therefore the MRJ state 12]. However, as pointed out in Ref. [12], a substantial hurdle to be overcome is the necessity to generate all possible jammed states, or at least a representative sample of such states, in an unbiased fashion using a "universal" protocol in the large-system limit. Even if such a protocol could be developed, however, the issue of what weights to assign the resulting configurations remains. Moreover, there are other fundamental problems with entropic measures, as we discuss below, including its significance for two-dimensional monodisperse hard disk packings.

According to OSLN, maximally random is defined by "where the entropy of initial states is maximum" and imply that this is a universal measure of disorder. It is not clear exactly what the authors mean by entropy and how (or whether) it can be measured for "initial states". It is not obvious that one can relate the "randomness" of the final configurations (which is what OSLN are analyzing) to that of the initial configurations. It appears OSLN's rationale is that their algorithm goes to the "nearest" energy minimum from a given initial configuration. Does this process preserve "entropy" or randomness? Clearly if one used, for example, global energy minimization, one would obtain very different results. Furthermore, entropy is a concept inherently related to distributions of configurations. However, one classifies particular final configurations (packings) as random or disordered, and by considering a given configuration, one can devise a procedure for quantitatively measuring (using order metrics) how disordered or ordered it is. This distinction between distributions of configurations and particular configurations is an important one that OSLN do not make.

The MRJ state is defined in [1] as the jammed state which minimizes a given order metric $\psi$. OSLN suggest their interpretation of maximally random as superior because using order metrics "will always be subject to uncertainty since one never knows if one has calculated the proper order parameter." Therefore, OSLN believe that 
they have identified the proper, unique, measure of order (related to entropy). We wish to stress the difference between well-defined and unique, as the two seem to be blurred in Ref. 4]. The MRJ state is well-defined in that for a particular choice of jamming category and order metric it can be identified unambiguously. For a finite system, it will consist of a discrete set (possibly one) of configurations, becoming more densely populated as the system becomes larger. At least for collective and strict jamming in three dimensions, a variety of sensible order metrics seem to produce an MRJ state near $\phi \approx 0.64$ [12], the traditionally accepted density of the RCP state.

However, the density of the MRJ state should not be confused with the MRJ state itself. It is possible to have a rather ordered packing at this very same density; for example, a jammed but diluted vacancy FCC lattice packing [12]. This is why the two-parameter description of packings in terms of the density $\phi$ and order metric $\psi$, as in Ref. [1], is not only useful, but actually necessary.

OSLN's description of order implies a direct relation between probability densities and randomness, i.e., that the most probable 13] configurations represent the most disordered state. In this sense, one expects that the density of jammed configurations, when viewed as a threedimensional plot over the $\phi-\psi$ plane will be very strongly peaked around the MRJ point for very large systems, just as the probability distribution curves in Fig. 6 in Ref. [4] are very peaked around $\phi \approx 0.64$. As OSLN suggest themselves, this might explain why several different packing procedures yield similar hard-particle packings under appropriate conditions, historically designated as RCP.

However, this is far from being a closed question 14]. Consider two-dimensional monodisperse circular disk packings as an example. It is well-known that twodimensional analogs of three-dimensional computational and experimental protocols that lead to putative RCP states result in disk packings that are highly crystalline, forming rather large triangular domains (grains) [15]. Because such highly ordered packings are the most probable for these protocols, OSLN's entropic measure would identify these as the most disordered, a dubious proposition. An appropriate order metric, on the other hand, is capable of identifying a particular configuration (not an ensemble of configurations) of considerably lower density (e.g., a jammed diluted triangular lattice) that is consistent with our intuitive notions of maximal disorder. However, typical packing protocols would almost never generate such disordered disk configurations because of their inherent bias toward undiluted crystallization. This brings us to OSLN's claim that they have devised an unbiased universal protocol, to which we now turn our attention.

\section{UNIVERSAL, HARD AND SOFT ALGORITHMS}

In this section, we focus on the algorithms used by OSLN and point out why they are neither universal nor superior to other procedures. We point out the close relations between OSLN's algorithm for generating configurations near the onset of jamming and the Zinchenko hard-sphere packing algorithm [16]. Furthermore, we question OSLN's implication that using one kind of interaction potential (with three different exponents) and one algorithm amounts to exploring the space of all jammed configurations in an unbiased manner. This puts into doubt the claimed universality of the point $J$.

By fixing the interaction potential, initial density and energy minimization (conjugate gradient) algorithm, OSLN obtain a well-defined collection of final configurations with well-defined (not unique) properties. In essence, OSLN make their algorithm devoid of tunable parameters by simply choosing specific and fixed values for them. Both the Zinchenko and OSLN algorithms are "dynamics independent", in the sense that there is no tunable parameter for the rate of compression, which would be an analog of the cooling or quenching rate in molecular systems. Both also imply that this makes their algorithm universal or superior to other algorithms and that the (well-defined) results they obtain are somehow special. Most sensible algorithms will in fact produce a welldefined density in the limit of large systems given a choice of algorithmic parameters. For example, by changing the expansion rate in the Lubachevsky-Stillinger algorithm, one can achieve final densities for spheres anywhere in the range from 0.64 (fast expansion) to 0.74 (very slow expansion), as clearly illustrated in Fig. 2a in Ref. [1]. Therefore, if we followed the logic of OSLN, we could claim that any number in that range represents a special point. In our opinion, a good packing algorithm should be capable of generating a variety of packings, in both density and the amount of order. How can one ascertain that the packings one produces are "most random" if there are no other jammed packings to compare to?

OSLN use two main procedures to generate final configurations. The first procedure is to choose a density and then use conjugate gradients to find a nearby energy minimum, starting from a randomly-generated initial configuration $(T=\infty)$, as described in Section IIA in Ref. 4]. Using this procedure, OSLN sampled inherent structures [17] at fixed density to measure the fraction $f_{j}(\phi)$ of states that had nonzero bulk and shear moduli, and showed that $f_{j}$ has a strong system-size dependence with its derivative becoming a delta-function in the large system limit. It is important to note that this procedure as such has little or nothing to do with hard-sphere packings, especially for the kind of soft potentials $(\alpha \geq 3 / 2)$ that OSLN study. Many stable energy minima will be completely unrelated to packings, and especially not to those designated as MRJ states.

OSLN used a second procedure to study the mechani- 
cal and structural properties of systems near the onset of jamming $\phi_{c}$. In this procedure, a configuration is compressed (or decompressed) using very small steps in density until the bulk and shear moduli vanished (or nonzero moduli develop), as described in Section IIB in Ref. 4]. We now demonstrate that this procedure is closely related to Zinchenko's algorithm [16] for generating hard-sphere packings. Start at low density with a set of nonoverlapping spheres of diameter $\sigma$. Both algorithms then slowly grow the particles (OSLN in small increments, Zinchenko continuously) while moving the particles to avoid overlap [18]. In the Zinchenko algorithm, one strictly maintains the contact between particles as soon as they touch, which requires solving a system of ODE's containing the rigidity matrix of the packing 3 ] to find the necessary particle displacements. OSLN on the other hand, use conjugate gradients $(\mathrm{CG})$ to reminimize the potential energy, which will simply push the particles just enough to keep them nonoverlapping, i.e., almost in contact. This procedure continues in both algorithms until no further densification is possible without inducing overlap.

Accordingly, it is not surprising the packing configurations close to $\phi_{c}$ obtained in Ref. 4] closely resemble (in packing fraction, amorphous character, coordination, etc.) packings generated via a variety of bonafide hard-sphere algorithms (and experiments [19]). In particular, very similar packings are produced with the Lubachevsky-Stillinger (LS) algorithm [20, 21] (with sufficiently high expansion rates) and the Zinchenko algorithm [16]. OSLN criticize the LS algorithm for changing the density in a dynamic fashion. The stated advantage of the OSLN protocol is that one can "quench the sys- tem to the final state within a fixed energy landscape" since "the density is always held constant". We are very puzzled by this last claim in light of their admission (in Section IIB of Ref. 4]) that they slowly change the density of the packing to find $\phi_{c}$. In fact, OSLN do not seem to clearly distinguish between the two rather different procedures they employ: the first for finding inherent structures (at a fixed density) and the second for generating packings at the jamming threshold (which searches in density). Fig. 6 of Ref. [4], which supposedly represents the distributions of jamming thresholds $\phi_{c}$, defined by the second procedure, is obtained by differentiating the distribution generated with the first procedure, with no clear justification.

Most problematic of all is OSLN's claim that their results are universal. Despite the statement that "Starting with randomly generated $T=\infty$ states guarantees that we sample all [emphasis added] phase space equally", all that their first algorithm manages to explore is the space of energy minima for the particular chosen interaction potential. By comparing three different exponents $\alpha$, OSLN conclude that the exact form of the potential is not important. However, a much more convincing picture would have been made if they instead tried qualitatively different kinds of interaction potentials, rather then simply changing the curvature of the potential at the contact point. Otherwise, why focus on continuous interaction potentials at all? Since it is geometry (i.e., the nonoverlap condition on the spherical cores) that is crucial, the hard-sphere system offers a far "cleaner" system to study when trying to understand the special point $J$.
[1] S. Torquato, T. M. Truskett, and P. G. Debenedetti, Phys. Rev. Lett. 84, 2064 (2000).

[2] S. Torquato and F. H. Stillinger, J. Phys. Chem. B 105, 11849 (2001).

[3] A. Donev, S. Torquato, F. H. Stillinger, and R. Connelly, J. Comp. Phys. (2003), in press.

[4] C. S. O'Hern, E. Silbert, A. J. Liu, and S. R. Nagel, Phys. Rev. E. 68, 011306 (2003).

[5] The present Comment focuses on Section II of OSLN.

[6] R. Connelly, Structural Topology 14, 43 (1988).

[7] R. Connelly, K. Bezdek, and A. Bezdek, Discrete and Computational Geometry 20, 111 (1998).

[8] A. Donev, S. Torquato, F. H. Stillinger, and R. Connelly, J. App. Phys. 95, 989 (2004).

[9] R. Connelly and W. Whiteley, SIAM Journal of Discrete Mathematics 9, 453 (1996).

[10] R. Connelly, Invent. Math. 66, 11 (1982).

[11] Some of these can be found on our webpage 22].

[12] A. R. Kansal, S. Torquato, and F. H. Stillinger, Phys. Rev. E 66, 041109 (2002).

[13] Given a uniform (unbiased) sampling of the space of jammed configurations, as OSLN repeatedly stress.
[14] More fundamental questions have not yet been settled. For example, should one really ignore dynamics when trying to account for the well-known density of 0.64 ?

[15] OSLN's procedure is not immune to this tendency to crystallize, which is the reason why they chose to study bidisperse disk packings instead of monodisperse disk packings.

[16] A. Zinchenko, J. Comp. Phys. 114, 298 (1994).

[17] F. H. Stillinger and T. A. Weber, J. Chem. Phys. 83, 4767 (1985).

[18] OSLN sometimes use the reverse procedure in which they decrease the density, which does not directly relate to the Zinchenko algorithm.

[19] G. D. Scott and D. M. Kilgour, J. Phys. D 2, 863 (1969).

[20] B. D. Lubachevsky and F. H. Stillinger, J. Stat. Phys. 60, 561 (1990), see also Ref. 21.

[21] B. D. Lubachevsky, F. H. Stillinger, and E. N. Pinson, J. Stat. Phys. 64, 501 (1991), second part of Ref. 20.

[22] A. Donev, http://atom.princeton.edu/donev/Packing homepage for the sphere packing project, with useful supplementary materials. 